\title{
Removing Noises Induced by Gamma Radiation in Cerenkov Luminescence Imaging Using a Temporal Median Filter
}

\author{
Xu Cao, ${ }^{1}$ Yang Li, ${ }^{1}$ Yonghua Zhan, ${ }^{1}$ Xueli Chen, ${ }^{1}$ Fei Kang, ${ }^{2}$ Jing Wang, ${ }^{2}$ and Jimin Liang ${ }^{1}$ \\ ${ }^{1}$ Engineering Research Center of Molecular and Neuro Imaging, Ministry of Education and School of Life Science and Technology, \\ Xidian University, Xian, Shaanxi 710071, China \\ ${ }^{2}$ Department of Nuclear Medicine, Xijing Hospital, Fourth Military Medical University, Xian, Shaanxi 710032, China
}

Correspondence should be addressed to Jimin Liang; jiminliang@gmail.com

Received 21 April 2016; Revised 19 July 2016; Accepted 3 August 2016

Academic Editor: Kuangyu Shi

Copyright ( $\odot 2016 \mathrm{Xu}$ Cao et al. This is an open access article distributed under the Creative Commons Attribution License, which permits unrestricted use, distribution, and reproduction in any medium, provided the original work is properly cited.

\begin{abstract}
Cerenkov luminescence imaging (CLI) can provide information of medical radionuclides used in nuclear imaging based on Cerenkov radiation, which makes it possible for optical means to image clinical radionuclide labeled probes. However, the exceptionally weak Cerenkov luminescence (CL) from Cerenkov radiation is susceptible to lots of impulse noises introduced by high energy gamma rays generating from the decays of radionuclides. In this work, a temporal median filter is proposed to remove this kind of impulse noises. Unlike traditional CLI collecting a single CL image with long exposure time and smoothing it using median filter, the proposed method captures a temporal sequence of CL images with shorter exposure time and employs a temporal median filter to smooth a temporal sequence of pixels. Results of in vivo experiments demonstrated that the proposed temporal median method can effectively remove random pulse noises induced by gamma radiation and achieve a robust CLI image.
\end{abstract}

\section{Introduction}

Cerenkov luminescence imaging (CLI) has the ability to optically visualize radioactive decay signals from medical isotopes using optical imaging instruments and attracts more and more attention. CLI utilizes a type of electromagnetic radiation called Cerenkov radiation produced when a charged particle travels faster than the speed of light through an insulating medium [1,2]. CLI collects molecular information by detecting Cerenkov luminescence (CL) in a continuous spectrum from the ultraviolet through the visible spectrum emitted during Cerenkov radiation. CLI can offer Cerenkov radiations of many clinically available radionuclides for positron emission tomography (PET) and single photon emission computed tomography (SPECT) with relatively lower cost optical devices. Compared to PET and SPECT, CLI has the potential for bridging the information acquired from nuclear and optical imaging.

Robertson et al. first performed CLI on mice using positron-emitting radiotracers [3]. Furthermore, several research groups independently showed the feasibility of CLI for disease targeting and drug tracking in small animals [49]. Using an optical tomographic reconstruction method, researchers have obtained tomographic images using CL termed as Cerenkov luminescence tomography (CLT) [1015]. Intraoperative or endoscopic imaging based on $C L$ is also reported [16-19]. It is very encouraging that CLI has been used for clinical imaging of human body $[20,21]$.

However, the very weak CL from Cerenkov radiation of radionuclides is susceptible to high level of impulse noises introduced by high energy gamma rays. To obtain strong enough CL signals for imaging, traditional CLI collects one image of CL with long exposure time about several minutes. At the same time, a great number of gamma rays may reach the CCD sensor and produce heavy random impulse noises. So, using shorter exposure time is beneficial to reduce the random impulse noises induced by gamma radiation on the premise of obtaining observable CL signals.

In the current study, single CL image acquisition with minute-long exposure time is substituted by a temporal sequence of CL images with second-long exposure time to suppress these random impulse noises. Accordingly, a 


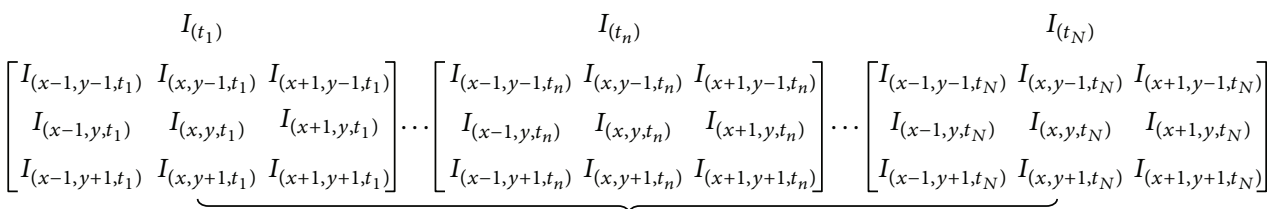

$$
\begin{aligned}
& \begin{array}{c}
\text { Median filter } \\
\downarrow \\
M_{(x, y)}
\end{array}
\end{aligned}
$$

FIGURE 1: Schematic diagram of temporal median filter.

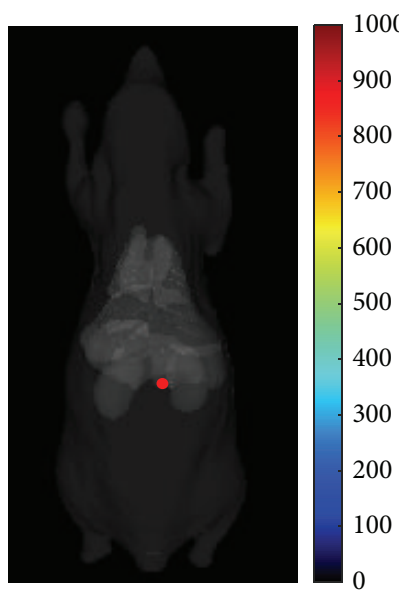

(a)

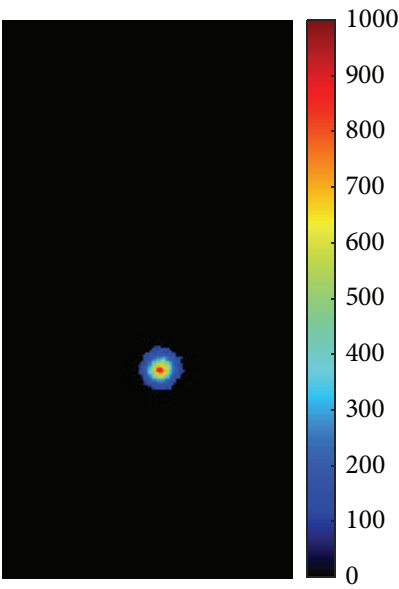

(b)

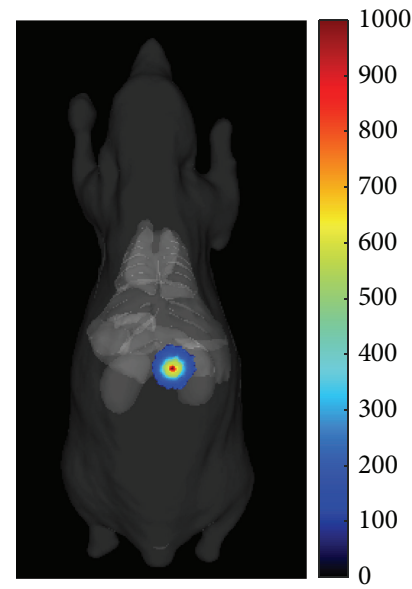

(c)

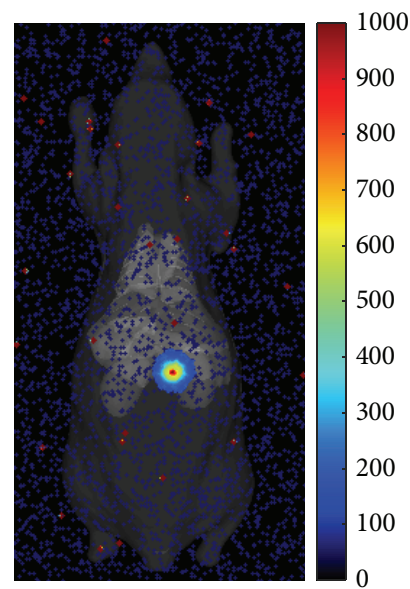

(d)

Figure 2: Numerical simulation of CL image. (a) The digital mouse and CL source location. (b) The simulated CLI image. (c) The fusion image of simulated CL and CT data. (d) The fusion image of simulated CL with impulse noises and CT data.

temporal median (TM) filter is proposed to remove the high level impulse noises induced by gamma radiation in CLI. Unlike traditional median filter smoothing pixels within spatial domain, TM filter smooths pixels within time domain, which is more effective to remove this kind of additive random noises.

\section{Material and Methods}

2.1. Temporal Median Filter. Figure 1 shows the scheme of TM filter. $N$ CLI images are consecutively collected, and $I_{\left(x, y, t_{n}\right)}$ is the gray value at pixel location $(x, y)$ for CLI image $I_{\left(t_{n}\right)}$ collected at $t_{n}$ time point.

We select 9 pixels containing eight neighbor pixels of $I_{\left(x, y, t_{*}\right)}$ and $I_{\left(x, y, t_{*}\right)}$ itself for each CLI image collected at $t_{*}$ time point, and then $9 N$ pixels for $N$ CLI images are used to construct a 1-D vector:

$$
U_{(x, y)}=\left[I_{\left(x-1, y-1, t_{1}\right)}, \ldots, I_{\left(x, y, t_{n}\right)}, \ldots, I_{\left(x+1, y+1, t_{N}\right)}\right]
$$

To get the median of $U_{(x, y)}$, we sort $U_{(x, y)}$ in ascending order and obtain a new $1-\mathrm{D}$ vector:

$$
R_{(x, y)}=\left[r_{(x, y, 1)}, \ldots, r_{(x, y, n)}, \ldots, r_{(x, y, 9 N)}\right]
$$

where $R_{(x, y)}\left(r_{(x, y, 1)} \leq \cdots \leq r_{(x, y, n)} \leq \cdots \leq r_{(x, y, 9 N)}\right)$ are the elements of $U_{(x, y)}$. The final filtered pixel value at location $(x, y)$ is the value at the middle position in $R_{(x, y)}$ :

$$
M_{(x, y)}=r_{(x, y,[9 N / 2\rceil)},
$$

where $\lceil 9 N / 2\rceil$ is the rounded up nearest integer of $9 N / 2 . N$ is the number of CLI images for a temporal sequence, which also means the sequence length in time domain.

2.2. Numerical Simulation. A Monte Carlo simulation using MOSE software based on digital mouse atlas is conducted to get the CL image. A point light source mimicking CL source generated from medical isotopes is subcutaneously embedded on back of the digital mouse with the depth about $5 \mathrm{~mm}$ as shown in Figure 2(a). The optical parameters $\mu \mathrm{a}$ and $\mu s$ of each organ under the wavelength of $620 \mathrm{~nm}$ are adopted from [22]. Figure 2(b) is the simulated CL image, and Figure 2(c) is the fused image with mouse atlas. Two levels of random noises were added into the CL image to simulate dark noises of CCD with low values and impulse noises induced by gamma radiation with high values (Figure $2(\mathrm{~d})$ ).

2.3. Materials. ${ }^{18}$ F-FDG was provided from the Department of Nuclear Medicine, Xijing Hospital, Fourth Military Medical University. Animal was cared for in accordance with a protocol approved by the Xidian University Animal Care and 


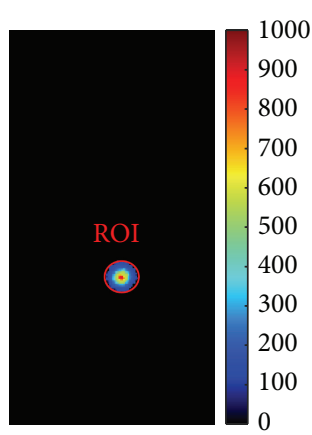

(a)

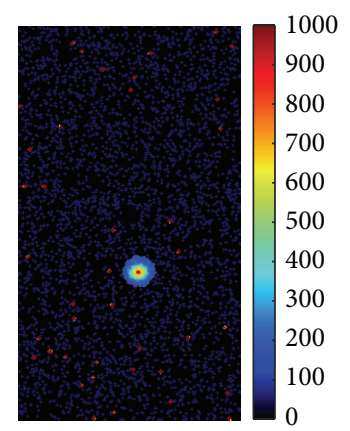

(b)

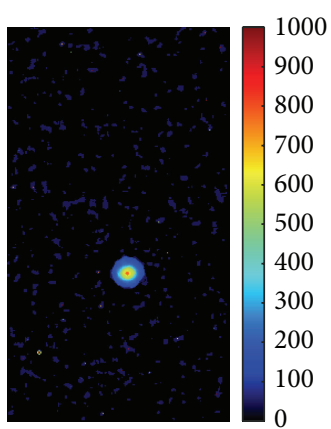

(c)

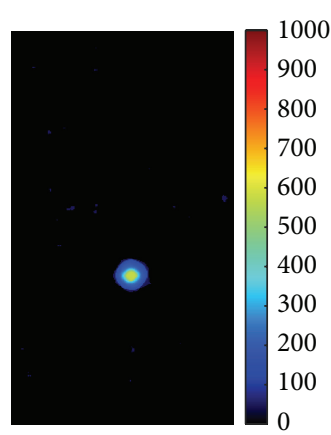

(d)

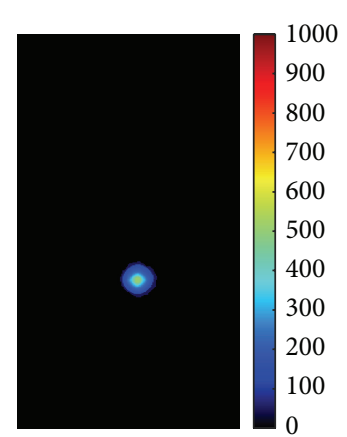

(e)

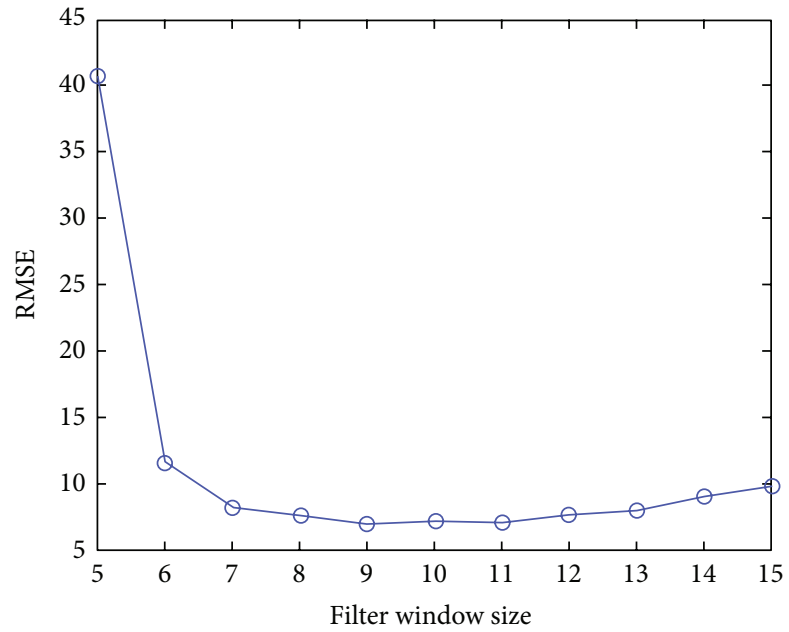

(f)

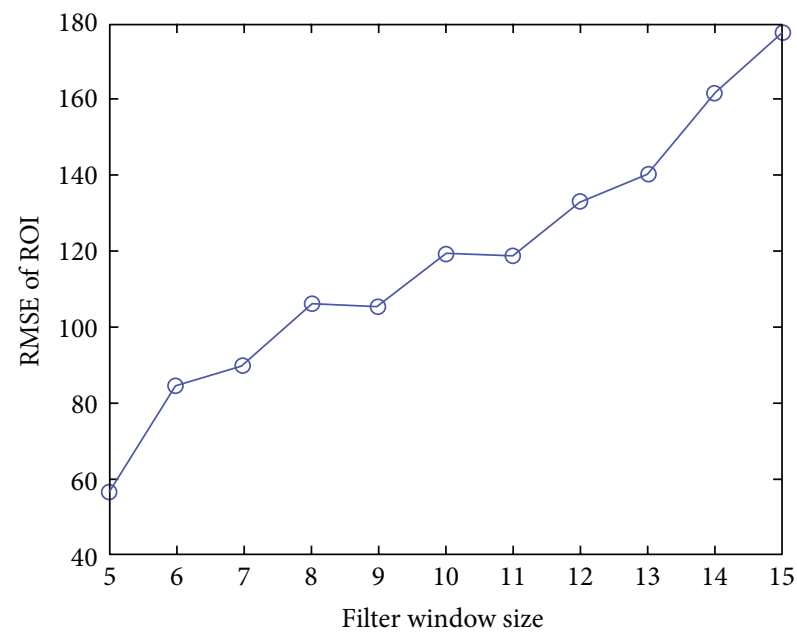

(g)

FIGURE 3: The traditional denoising method for simulated CLI. (a) Simulated CLI image. (b) Simulated CLI image with noises. (c-e) Filtered CLI images using median filter with filter window size $=5,10$, and 15. (f, g) Root mean squared error of the filtered CLI image and ROI of that.

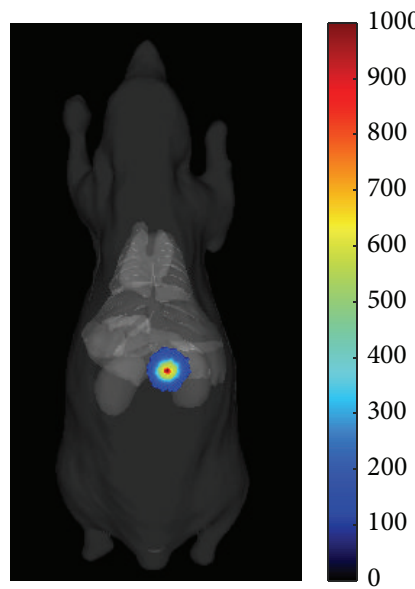

(a)

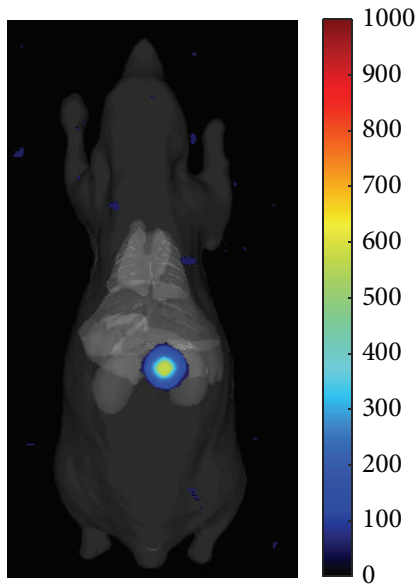

(b)

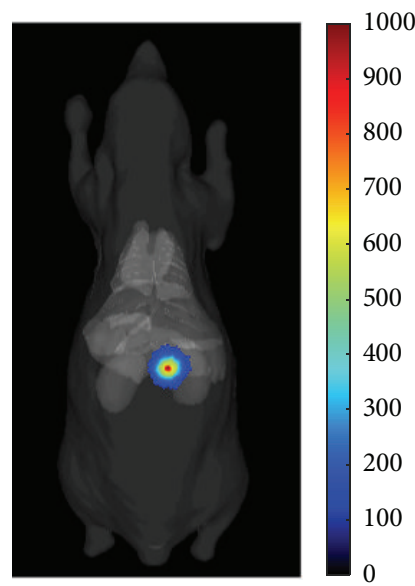

(c)

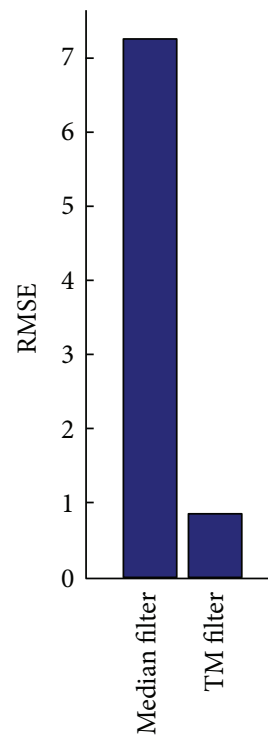

(d)

FIGURE 4: A comparison between median filter and TM filter. (a) CLI image with no noise. (b) Result of median filter. (c) Result of TM filter. (d) Comparison of RMSE for the two methods. 


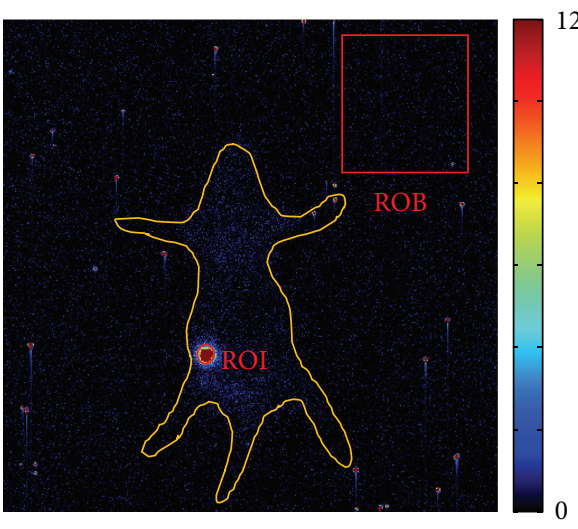

(a)

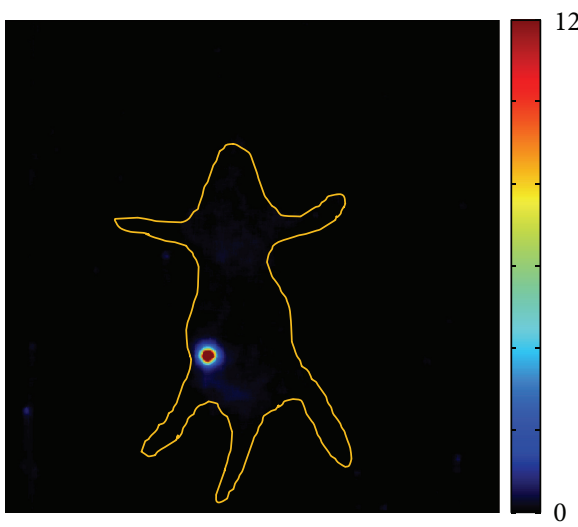

(c)

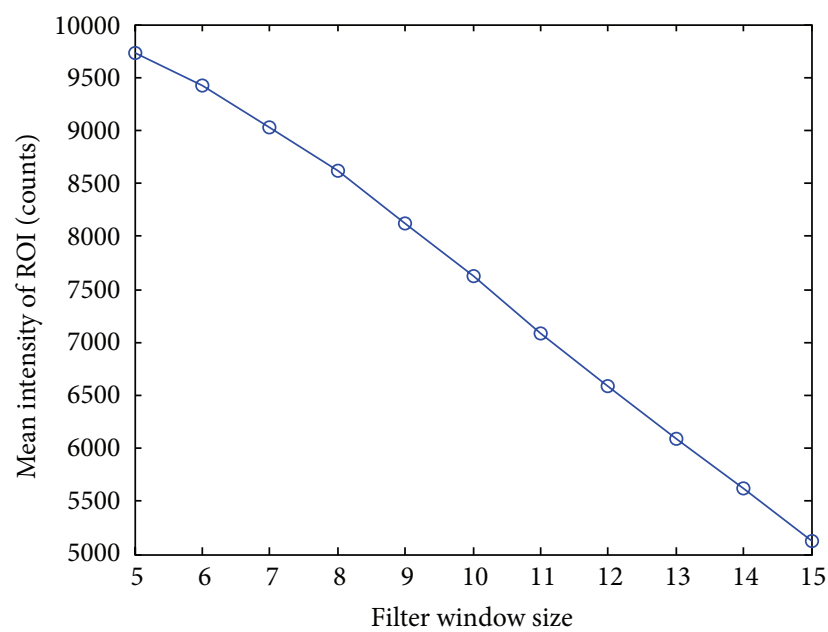

(e)

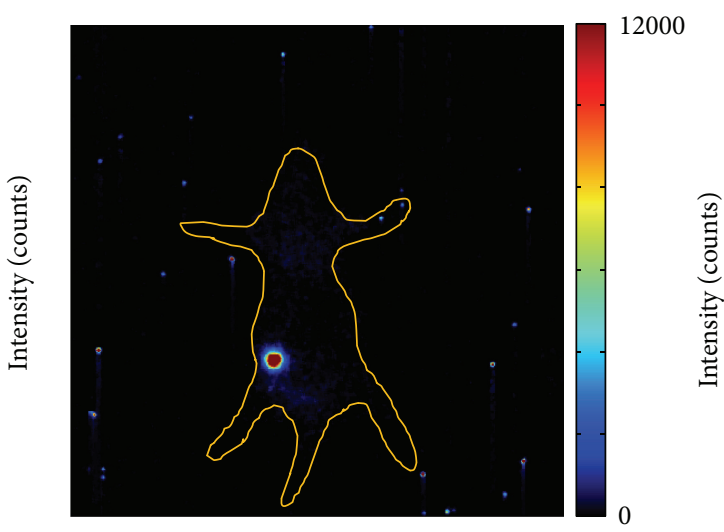

(b)

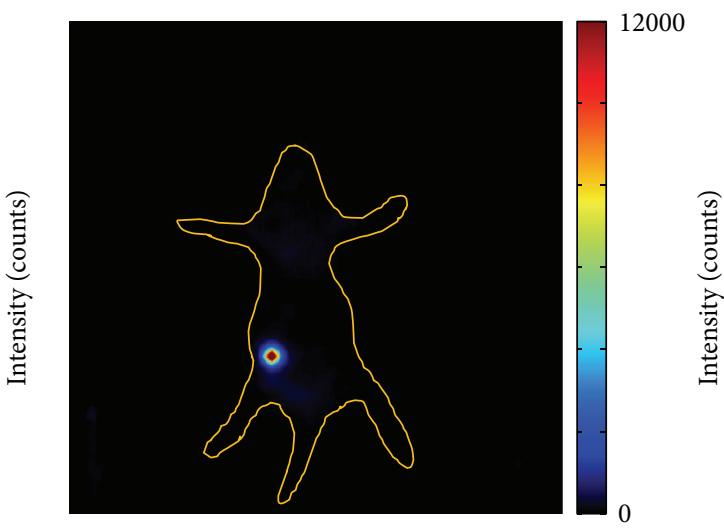

(d)

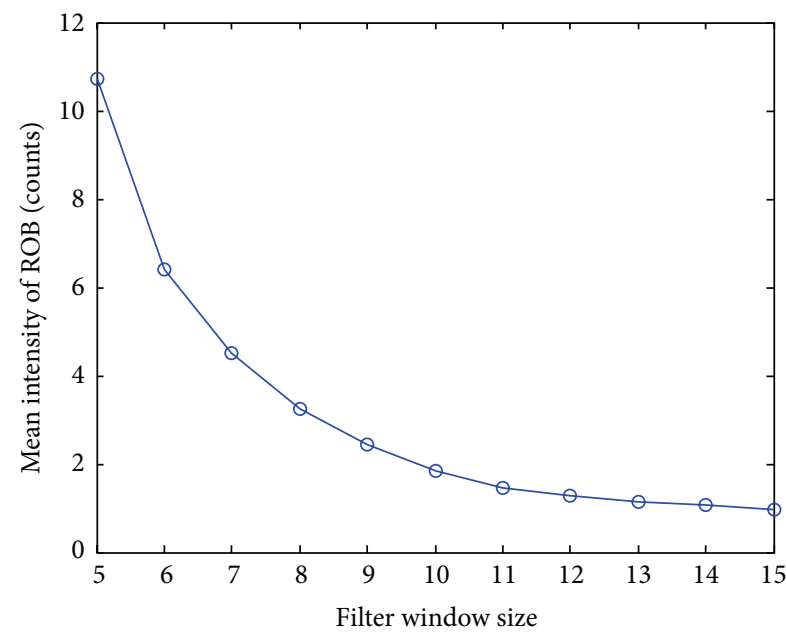

(f)

Figure 5: The traditional denoising method for CLI. (a) Original CLI image captured with 60 s exposure time. (b-d) Filtered CLI images using median filter with filter window size $=5,10$, and 15. A large filter window size achieves a smooth CLI image with less impulse noises. $(\mathrm{e}, \mathrm{f})$ Quantitative analysis of the relations between signal values in region of interest (ROI) marked with red circle and region of background (ROB) marked with red rectangle. The signal values in both ROI and ROB decrease with the increase of filter window size.

Use Committee. A Kunming mouse with abdomen unhairing using depilatory cream was used as the imaging object. A pseudotumor was provided using $100 \mathrm{uCi}$ of ${ }^{18} \mathrm{~F}-\mathrm{FDG}$ with the volume $20 \mathrm{uL}$ mixed with $20 \mathrm{uL}$ matrigel (BD Biosciences, Sparks, MD) injected to abdomen of the mouse.
2.4. Optical Imaging. All CLI images were acquired using a home-made in vivo animal optical imaging system, which includes an Andor Ixon Ultra 897 EMCCD with a Schneider $25 \mathrm{~mm} \mathrm{f/0.95} \mathrm{lens.} \mathrm{After} \mathrm{the} \mathrm{mouse} \mathrm{was} \mathrm{anesthetized} \mathrm{with}$ intraperitoneal injection of $100 \mathrm{uL}$ anesthetic, the mouse was 


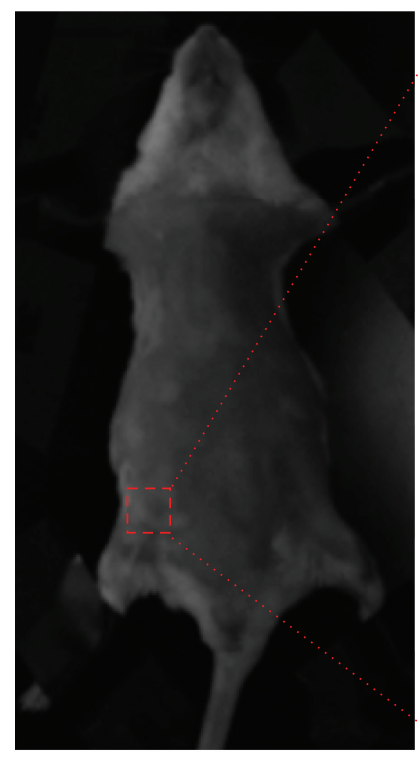

(a)

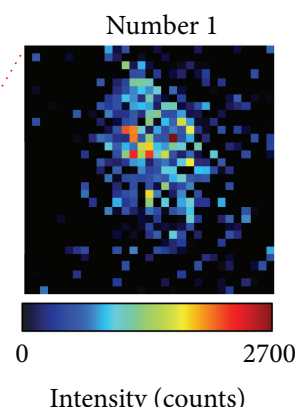

Intensity (counts)

Number 16

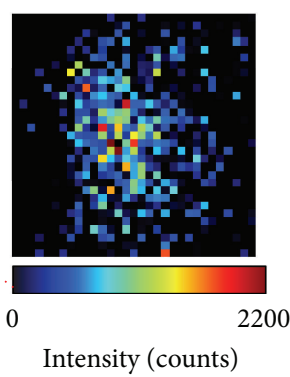

Number 6

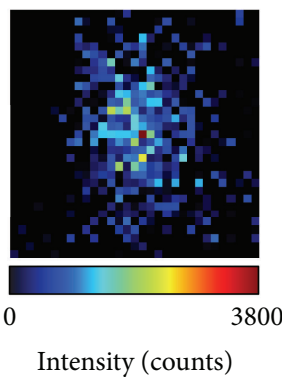

Number 21

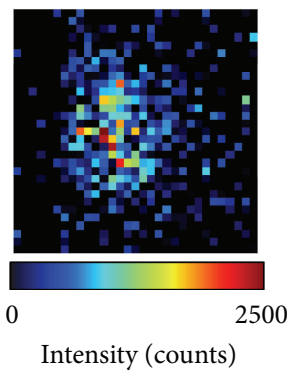

(b)

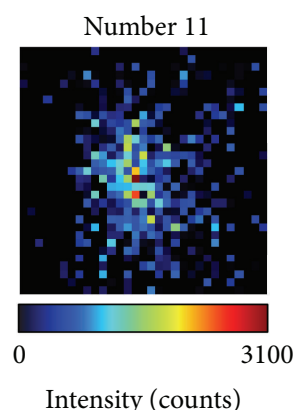

Number 26

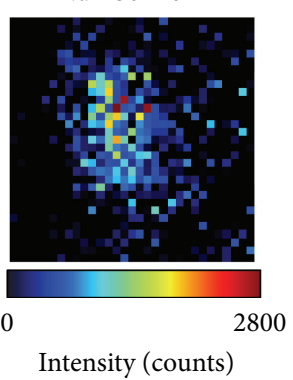

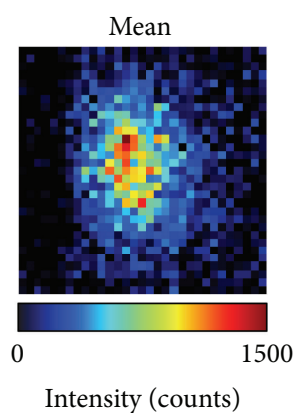

(c)

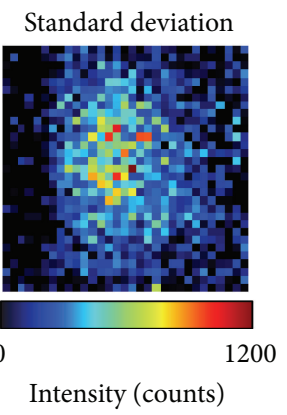

(d)

FIGURE 6: Original CLI images and mean and standard deviation of a CLI image sequence containing 30 CLI images with exposure time of $10 \mathrm{~s}$. (a) is the photograph of the mouse from supine view, and the red dashed box indicates the location of pseudotumor. (b) is detailed view of red dashed box for number 1, 6, 11, 16, 21, and 26 CLI images. (c) is mean image of the CLI image sequence. (d) is the standard deviation image for the CLI image sequence.

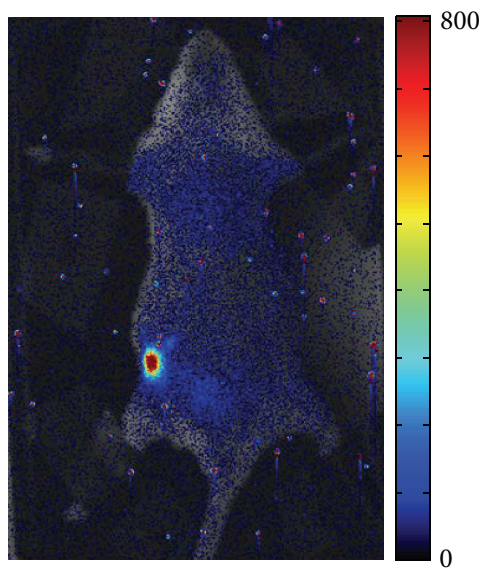

(a)

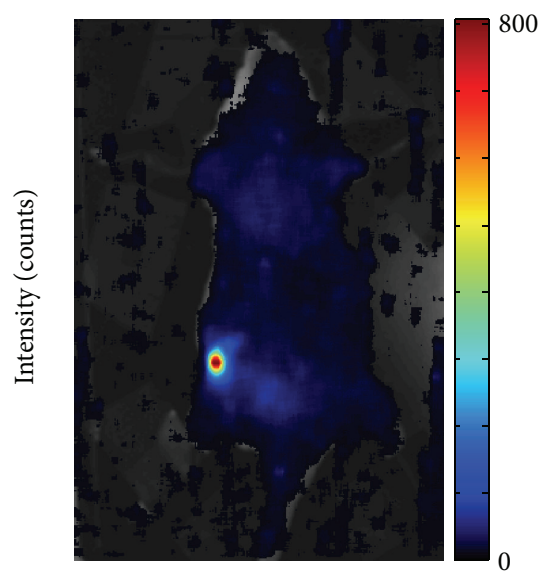

(b)

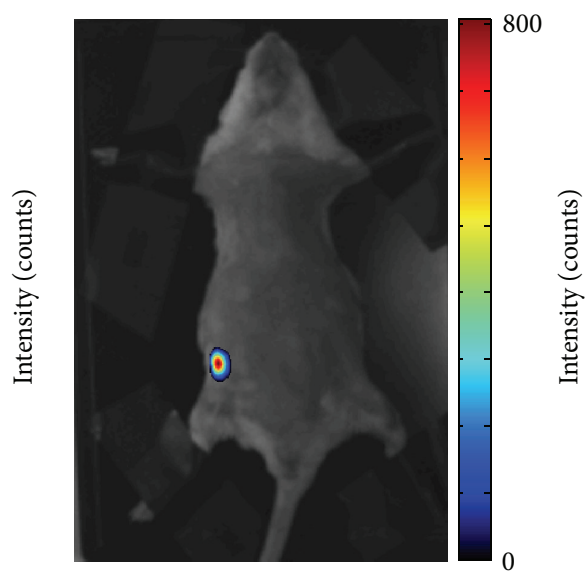

(c)

Figure 7: A randomly chosen original CLI image from a sequence of 30 CLI images with exposure time of $10 \mathrm{~s}$ for each one (a), result of median filter fused with white light image (b), and result of TM filter fused with white light image (c).

put into a light-tight chamber of the imaging system and the collection of CLI images started.

\section{Results and Discussion}

3.1. Results of Numerical Simulation. Figure 3(a) is the simulated CL image, and Figure $3(\mathrm{~b})$ is that with noises. The traditional median filter is usually used for removing these impulse noises [18, 20]. Figures 3(c)-3(e) are filtered CLI images using median filter with different filter window sizes. With the increase of filter window size, less noises remain in the filtered CLI image, but pixel values in region of interest (ROI) have more deviations. Root mean squared error (RMSE) of the filtered CLI image and ROI of that can clearly demonstrate this trend. It means that the median filter can effectively filter out impulse noises but can introduce 


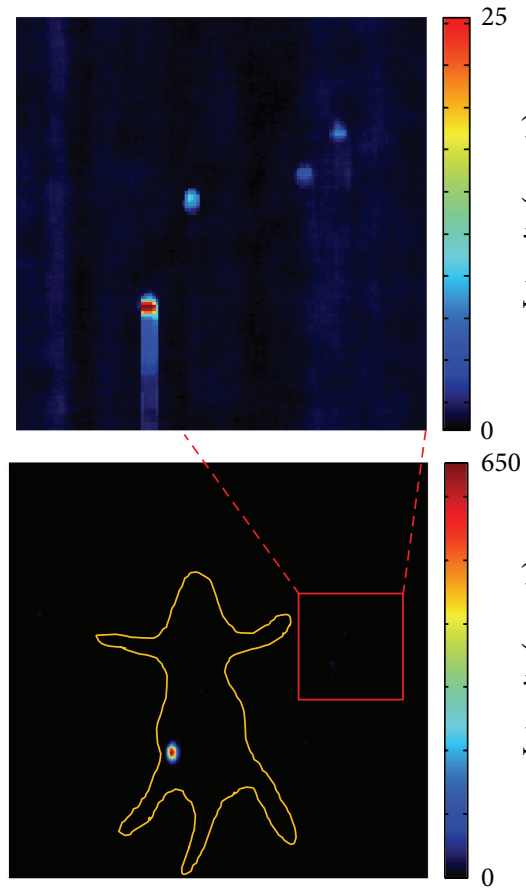

(a)

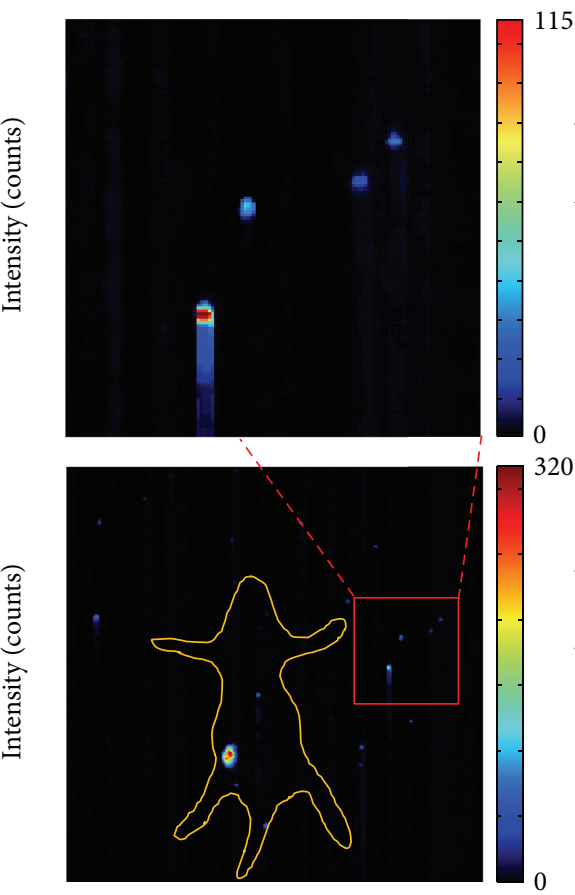

(b)

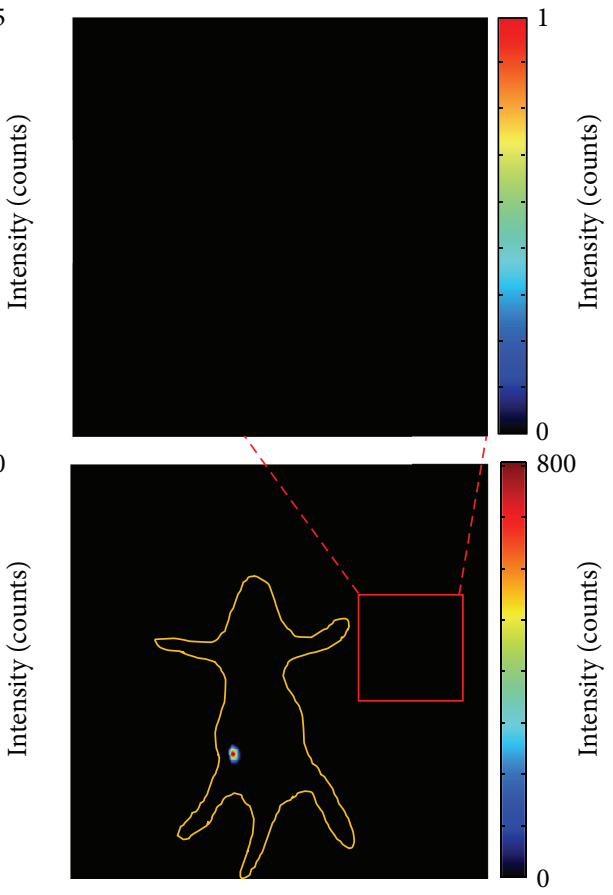

(c)

Figure 8: Mean image of 30 CLI images filtered using median filter (a), standard deviation image of 30 CLI images filtered using median filter (b), and filtered CLI image using TM filter (c). The top is the enlarged image corresponding to the red rectangle region.
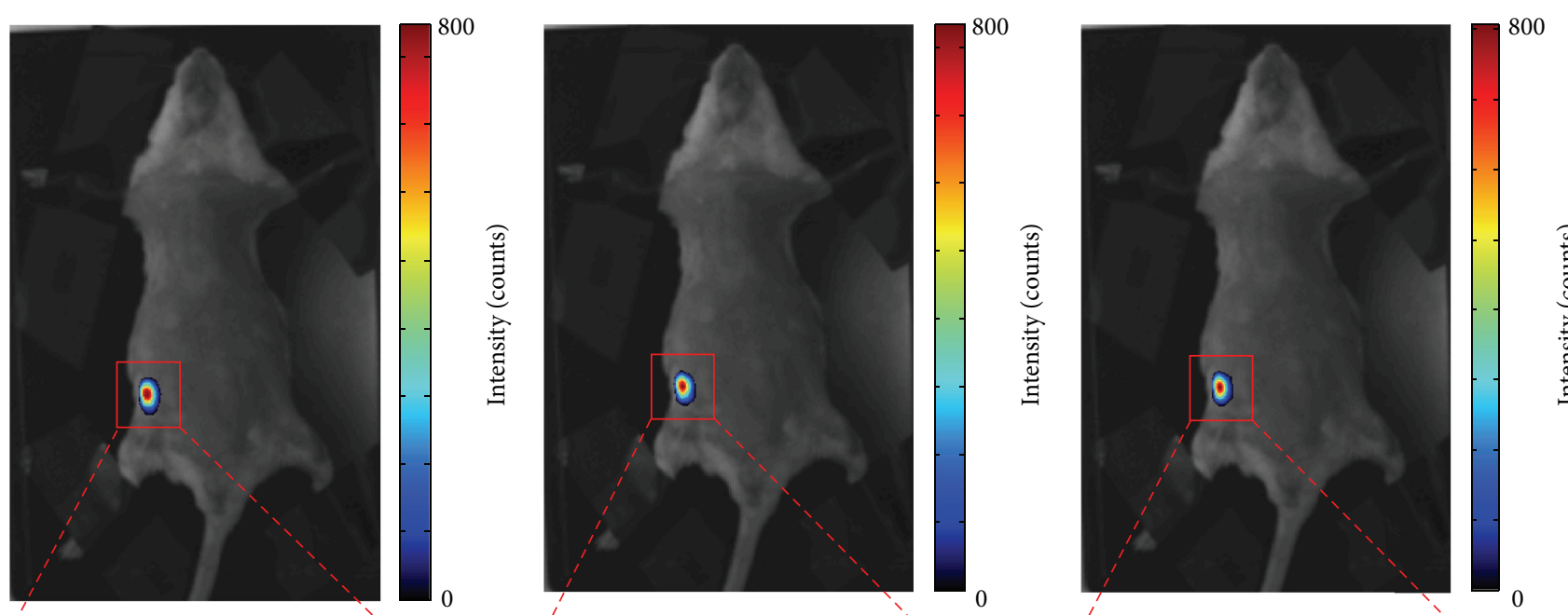

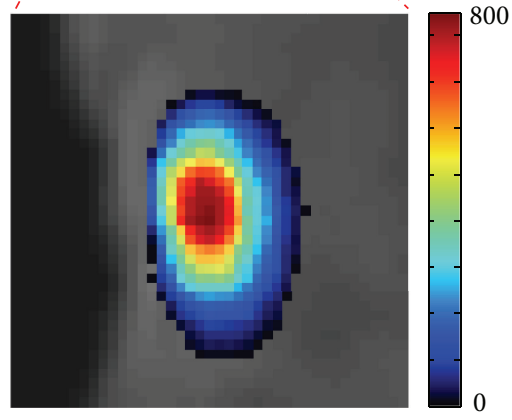

(a)

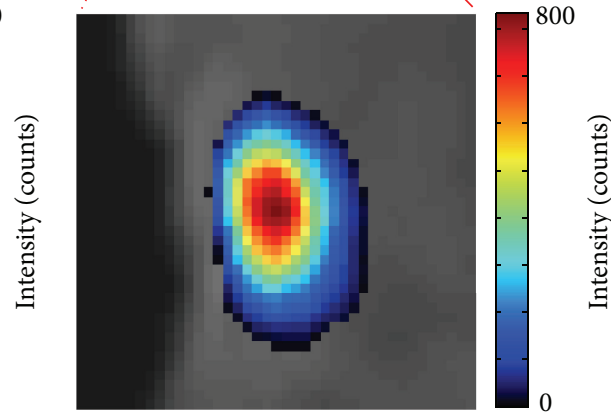

(b)

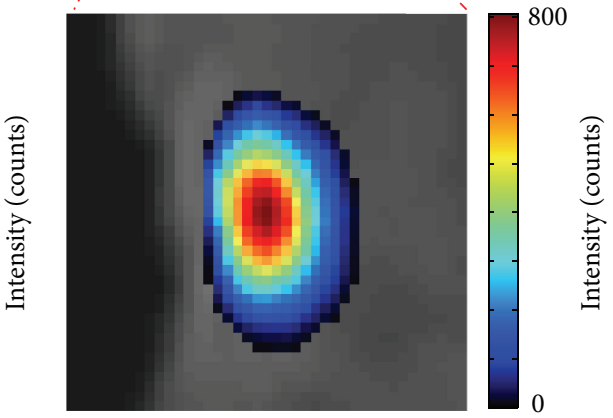

(c)

FIGURE 9: The influence of sequence length to TM filter. (a), (b), and (c) are results of TM filter for $N=5, N=10$, and $N=30$, respectively. 

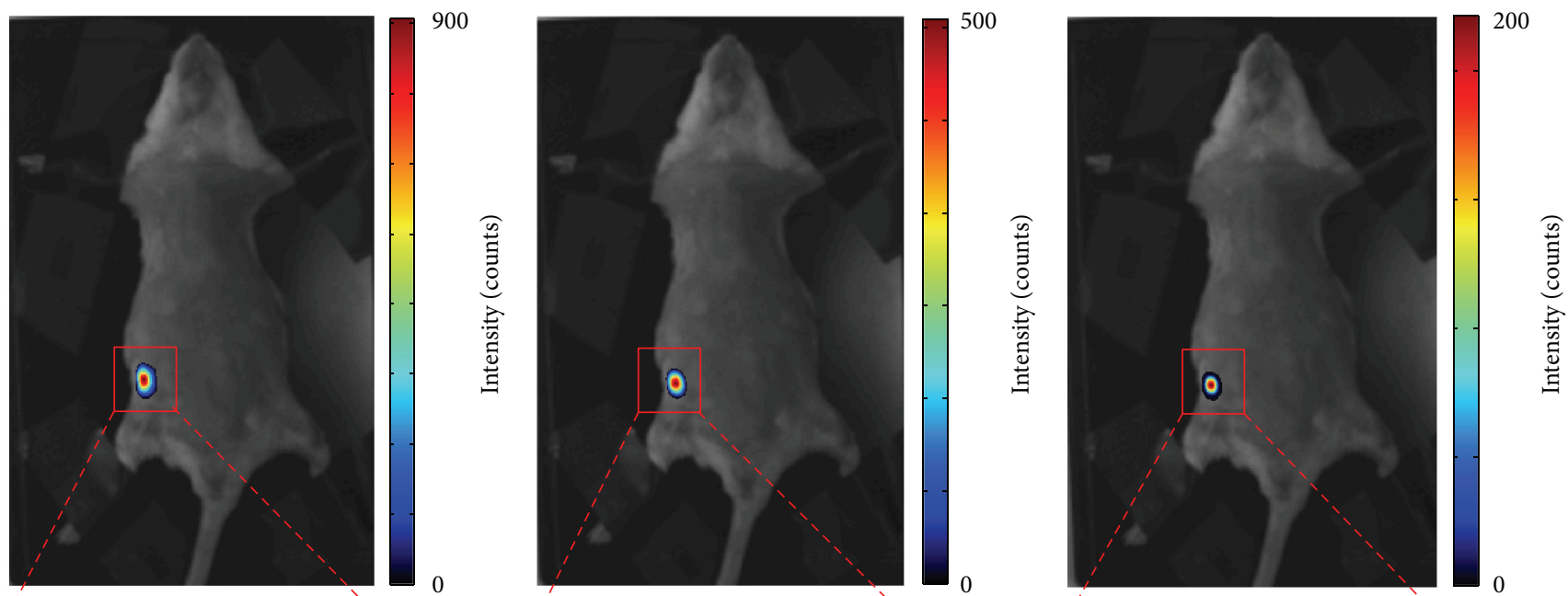

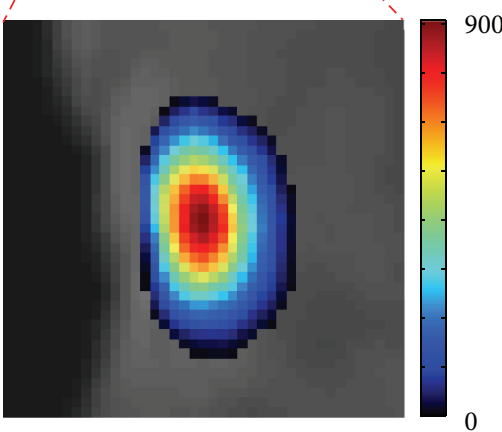

(a)

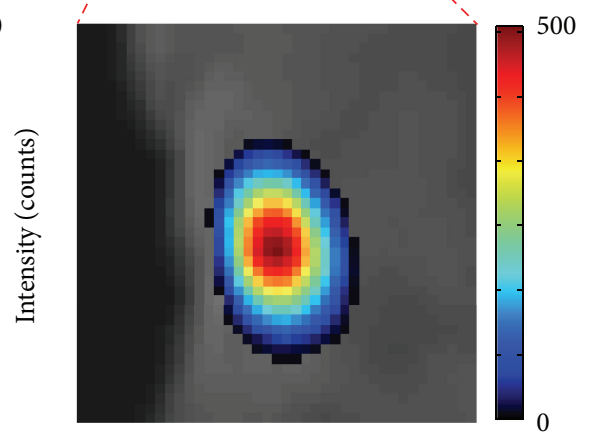

(b)

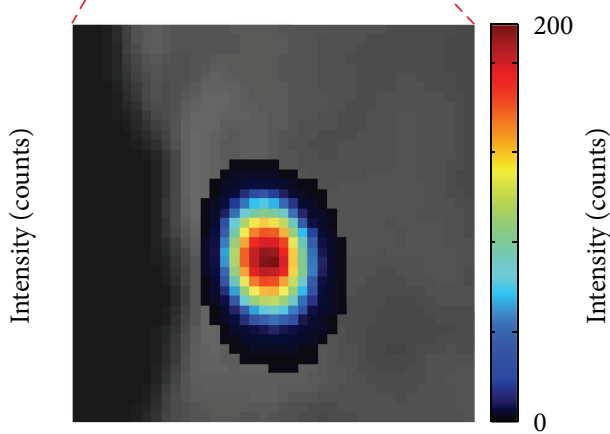

(c)

Figure 10: The results for TM filter with different exposure time $T$. (a), (b), and (c) are $T=10 \mathrm{~s}, T=5 \mathrm{~s}$, and $T=2 \mathrm{~s}$, respectively.

large errors for ROI of CL signals, when large filter window size is employed. On the contrary, the median filter with small filter window can keep ROI of filtered CLI image with the same value as that of simulated CL image, but with lots of impulse noises.

Figure 4 summarizes the results of median filter and TM filter for numerical simulations. Result of median filter has a few low background noises and large deviation in ROI. Quantified analysis using RMSE for the two methods has also illustrated that TM filter can acquire a high quality image for CLI.

3.2. Impulse Noises in CLI Image. CL signals are so weak that a long exposure time is necessary for CLI. During the long exposure process, a great number of gamma rays may reach CCD sensor and produce heavy random impulse noises. Figure 5(a) is an original CLI image captured with $60 \mathrm{~s}$ exposure time. Although ${ }^{18} \mathrm{~F}-\mathrm{FDG}$ locates in region of the pseudotumor (ROI marked with red circle), lots of impulse noises intersperse among other locations in and out of the mouse without ${ }^{18} \mathrm{~F}$-FDG as a result of the reach of gamma rays with arbitrary directions. Figures 5(b)-5(d) are filtered CLI images using median filter with different filter window sizes. The filtered CLI image with small filter window size contains lots of noises but the pixel values in region of signal are close to that of the original CLI image
(Figure 5(b)). While a large filter window size can obtain an enough smooth filtered CLI image, the pixel values in region of signal are much smaller than that of the original CLI image (Figure 5(d)). Figures 5(e) and 5(f) are the quantified results of pixel values in ROI marked with red circle and ROB marked with red rectangle in the original CLI image.

Figure 6 shows CLI images of pseudotumor in the mouse with a short exposure time of $10 \mathrm{~s}$ captured at different time points. From 6 selective CLI images, we can see that the distributions of CL are generally in agreement, but differences exist in the details. The mean and standard deviation images can further illustrate these differences. The difference in capture time is not the main reason, because the interval time between each CLI image is less than $1 \mathrm{~s}$. The fast gelforming BD matrix can prevent ${ }^{18}$ FDG diffusing. So the high level impulse noises produce these differences. The standard deviation image also shows that the impulse noises are almost the same level as the CL signals.

3.3. Result of TM Filter. A sequence of 30 CLI images with exposure time of $10 \mathrm{~s}$ for each one was used to test TM filter. To compare with median filter, we randomly chose a CLI image from the sequence shown in Figure 7(a). It is clearly seen that random impulse noises induced by gamma radiation have higher intensities than that of CL signals from 
high ${ }^{18}$ FDG concentration area. Figure 7(b) is the result of median filter, which has lots of low background noise that emerged with the smooth process of median filter. The result of TM filter almost has no noise shown in Figure 7(c).

To further illustrate noise suppression of TM filter, we investigate the background region outside of the mouse after filtering. If TM filter is effective for suppressing these impulse noises, there will be no noises in the background region of the filtered CL image. Due to median filter works on a single CLI image, we obtain mean and standard deviation images of 30 filtered CLI images using median filter as shown in Figures $8(\mathrm{a})$ and $8(\mathrm{~b})$ to compare with TM filter. In Figure 8(a), there seem to be no noises in the background region after average of 30 filtered CLI images using median filter, but noises can be clearly seen in the enlarged image of the region of background marked with red rectangle. More noises emerge from the standard deviation image of 30 filtered CLI images using median filter in Figure 8(b). The filtered CLI image using TM filter in Figure 8(c) almost has no impulse noises, and particularly we find no noise in the enlarged background image.

3.4. Sequence Length for TM Filter. Generally, a large sequence containing more CLI images has greater ability to remove random impulse noises, but that will cost more time for data acquisition. Figure 9 shows the influence of sequence length to the TM filter. The proposed TM filter can maintain the distributions and values of CL signals even when the number of CLI images decreases to $N=5$.

3.5. Exposure Time for Each CLI Image. Short exposure time is helpful in reducing the random pulse noise induced by gamma radiation, but CL signals cannot be collected using unduly short exposure time when radioactivity is rather low. In this experiment, the radioactivity of ${ }^{18} \mathrm{~F}$-FDG in the pseudotumor was $100 \mathrm{uCi}$. We use different lengths of exposure time to investigate the characteristic of TM filter. Figure 10 summarizes results of TM filter with exposure time of $T=10 \mathrm{~s}, T=5 \mathrm{~s}$, and $T=2 \mathrm{~s}$, respectively. The intensity and area of CL signals decrease with the exposure time $T$. Even though the exposure time $T$ is shortened to $2 \mathrm{~s}$, TM filter can still obtain a satisfactory result.

\section{Conclusions}

In this work, a TM filter is proposed to remove the random impulse noises induced by high energy gamma rays generated from radioactive decay of medical nuclides. This method synthesizes characteristics of randomness and pulse for random pulse noises in CL images and employs a temporal median-like filter in a temporal sequence of CL images. Several simulation and in vivo experiments were presented to verify the proposed TM filter, and these results demonstrated that TM filter can effectively remove random impulse noises induced by gamma radiation and achieve a robust CL image. Several key properties of TM filter were also investigated based on in vivo experimental data to fully understand robustness and usability of this method.
In terms of extremely weak CL signal, traditional CLI collects a single CL image with minutes-long exposure time, which often contains lots of random impulse noises due to the arrival of gamma rays to CCD sensor. With the increase of the exposure time, the original CL image contains more and more impulse noises. In order to filter out these random pulse noises, traditional median filter with a large filter window size is employed. The filtered CL image of this single acquisition strategy shows instability and oversmoothness. The proposed method can overcome these drawbacks by collecting a temporal sequence of CLI images with seconds-long exposure time and a TM filter. With a multiple CLI images strategy, the proposed method is useful for suppressing these random pulse noises and getting robust CLI image. The smooth effect of TM filter is to work on pixels of CLI images collected at different time points located in the same coordinate, so the proposed method avoids smoothing CLI image on spatial domain when removing the random pulse noises. Finally, the proposed method can acquire high quality CLI images with high robustness.

To concentrate on the validity of TM for CLI, we perform an in vivo experiment based on a pseudotumor model. The nonspecific distributions of medical nuclides often make the actual CLI applications contain complicated circumstances. So the proposed method needs to be tested by different actual CLI applications.

\section{Competing Interests}

The authors declare that they have no competing interests.

\section{Acknowledgments}

This work was supported by the Program of the National Natural Science Foundation of China under Grant nos. 81227901, 61405149, 61471279, 31371006, and 81571725, the Natural Science Basic Research Plan in Shaanxi Province of China under Grant no. 2015JZ019, the Beijing Municipal Natural Science Foundation under Grant no. 7142012, and the Fundamental Research Funds for the Central Universities.

\section{References}

[1] P. Čherenkov, "Visible Emission of Č lean liquids by action of $\gamma$ radiation," Doklady Akademii Nauk SSSR, vol. 2, pp. 451-454, 1934.

[2] P. A. Čerenkov, "Visible radiation produced by electrons moving in a medium with velocities exceeding that of light," Physical Review, vol. 52, no. 4, pp. 378-379, 1937.

[3] R. Robertson, M. S. Germanos, C. Li, G. S. Mitchell, S. R. Cherry, and M. D. Silva, "Optical imaging of Cerenkov light generation from positron-emitting radiotracers," Physics in Medicine and Biology, vol. 54, no. 16, pp. N355-N365, 2009.

[4] A. E. Spinelli, D. D’Ambrosio, L. Calderan, M. Marengo, A. Sbarbati, and F. Boschi, "Čerenkov radiation allows in vivo optical imaging of positron emitting radiotracers," Physics in Medicine and Biology, vol. 55, no. 2, pp. 483-495, 2010.

[5] H. Liu, G. Ren, Z. Miao et al., "Molecular optical imaging with radioactive probes," PLoS ONE, vol. 5, no. 3, Article ID e9470, 2010. 
[6] R. S. Dothager, R. J. Goiffon, E. Jackson, S. Harpstrite, and D. Piwnica-Worms, "Čerenkov radiation energy transfer (CRET) imaging: a novel method for optical imaging of PET isotopes in biological systems," PLoS ONE, vol. 5, no. 10, Article ID e13300, 2010.

[7] G. S. Mitchell, R. K. Gill, D. L. Boucher, C. Li, and S. R. Cherry, "In vivo Čerenkov luminescence imaging: a new tool for molecular imaging," Philosophical Transactions of the Royal Society A: Mathematical, Physical and Engineering Sciences, vol. 369, no. 1955, pp. 4605-4619, 2011.

[8] A. Ruggiero, J. P. Holland, J. S. Lewis, and J. Grimm, "Čerenkov luminescence imaging of medical isotopes," Journal of Nuclear Medicine, vol. 51, no. 7, pp. 1123-1130, 2010.

[9] M. A. Lewis, V. D. Kodibagkar, O. K. Öz, and R. P. Mason, “On the potential for molecular imaging with Čerenkov luminescence," Optics Letters, vol. 35, no. 23, pp. 3889-3891, 2010.

[10] C. Li, G. S. Mitchell, and S. R. Cherry, "Čerenkov luminescence tomography for small-animal imaging," Optics Letters, vol. 35, no. 7, pp. 1109-1111, 2010.

[11] Z. Hu, J. Liang, W. Yang et al., "Experimental Čerenkov luminescence tomography of the mouse model with SPECT imaging validation," Optics Express, vol. 18, no. 24, pp. 2444124450, 2010.

[12] Z. Hu, X. Ma, X. Qu et al., "Three-dimensional noninvasive monitoring iodine-131 uptake in the thyroid using a modified cerenkov luminescence tomography approach," PLoS ONE, vol. 7, no. 5, Article ID e37623, 2012.

[13] J. Zhong, J. Tian, X. Yang, and C. Qin, "Whole-body cerenkov luminescence tomography with the finite element $\mathrm{SP}_{3}$ method," Annals of Biomedical Engineering, vol. 39, no. 6, pp. 1728-1735, 2011.

[14] J. Tian, J. Zhong, C. Qin, X. Yang, S. Zhu, and X. Zhang, "Čerenkov luminescence tomography for in vivo radiopharmaceutical imaging," International Journal of Biomedical Imaging, vol. 2011, Article ID 641618, 6 pages, 2011.

[15] A. E. Spinelli, C. Kuo, B. W. Rice et al., "Multispectral Čerenkov luminescence tomography for small animal optical imaging," Optics Express, vol. 19, no. 13, pp. 12605-12618, 2011.

[16] J. P. Holland, G. Normand, A. Ruggiero, J. S. Lewis, and J. Grimm, "Intraoperative imaging of positron emission tomographic radiotracers using cerenkov luminescence emissions," Molecular Imaging, vol. 10, no. 3, pp. 177-186, 2011.

[17] H. Liu, C. M. Carpenter, H. Jiang et al., "Intraoperative imaging of tumors using cerenkov luminescence endoscopy: a feasibility experimental study," Journal of Nuclear Medicine, vol. 53, no. 10, pp. 1579-1584, 2012.

[18] X. Cao, X. Chen, F. Kang et al., "Performance evaluation of endoscopic Čerenkov luminescence imaging system: in vitro and pseudotumor studies," Biomedical Optics Express, vol. 5, no. 10, pp. 3660-3670, 2014.

[19] H. Hu, X. Cao, F. Kang et al., "Feasibility study of novel endoscopic Čerenkov luminescence imaging system in detecting and quantifying gastrointestinal disease: first human results," European Radiology, vol. 25, no. 6, pp. 1814-1822, 2015.

[20] A. E. Spinelli and F. Boschi, "Human Čerenkov imaging using ${ }^{18}$ F-FDG," Journal of Nuclear Medicine, vol. 55, no. 3, pp. 523533,2014

[21] A. E. Spinelli, M. Ferdeghini, C. Cavedon et al., "First human cerenkography," Journal of Biomedical Optics, vol. 18, no. 2, Article ID 020502, 2013.
[22] K. Liu, Y. Lu, J. Tian et al., "Evaluation of the simplified spherical harmonics approximation in bioluminescence tomography through heterogeneous mouse models," Optics Express, vol. 18, no. 20, pp. 20988-21002, 2010. 

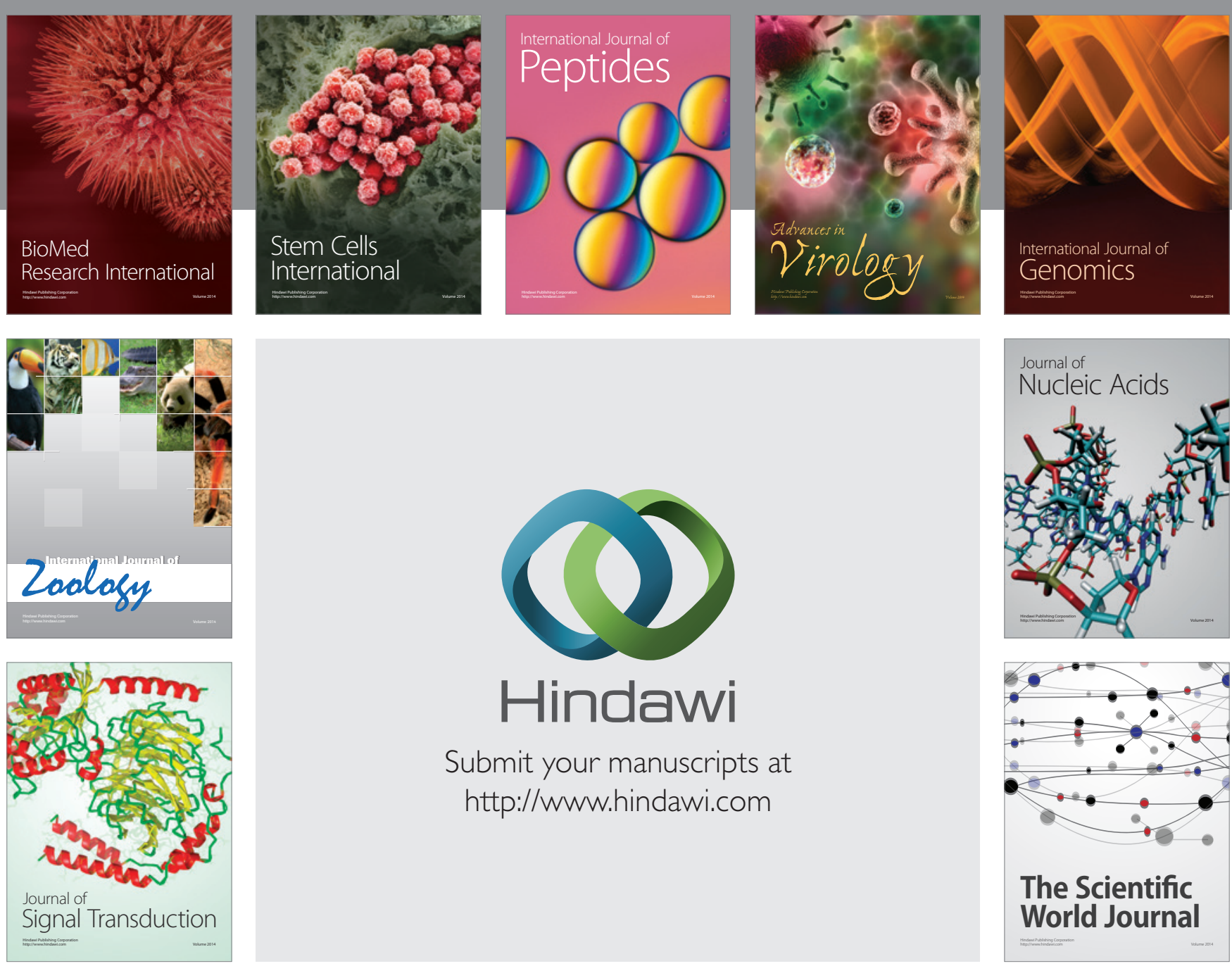

Submit your manuscripts at

http://www.hindawi.com
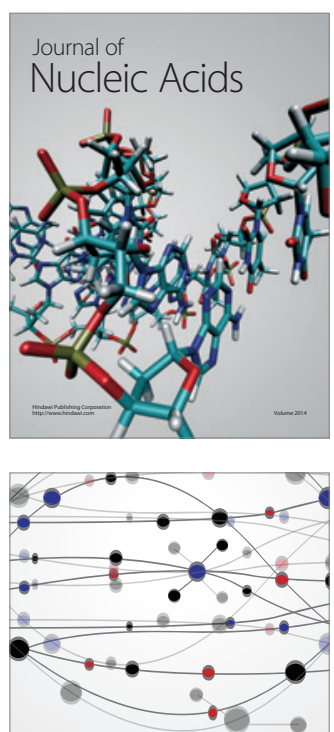

The Scientific World Journal
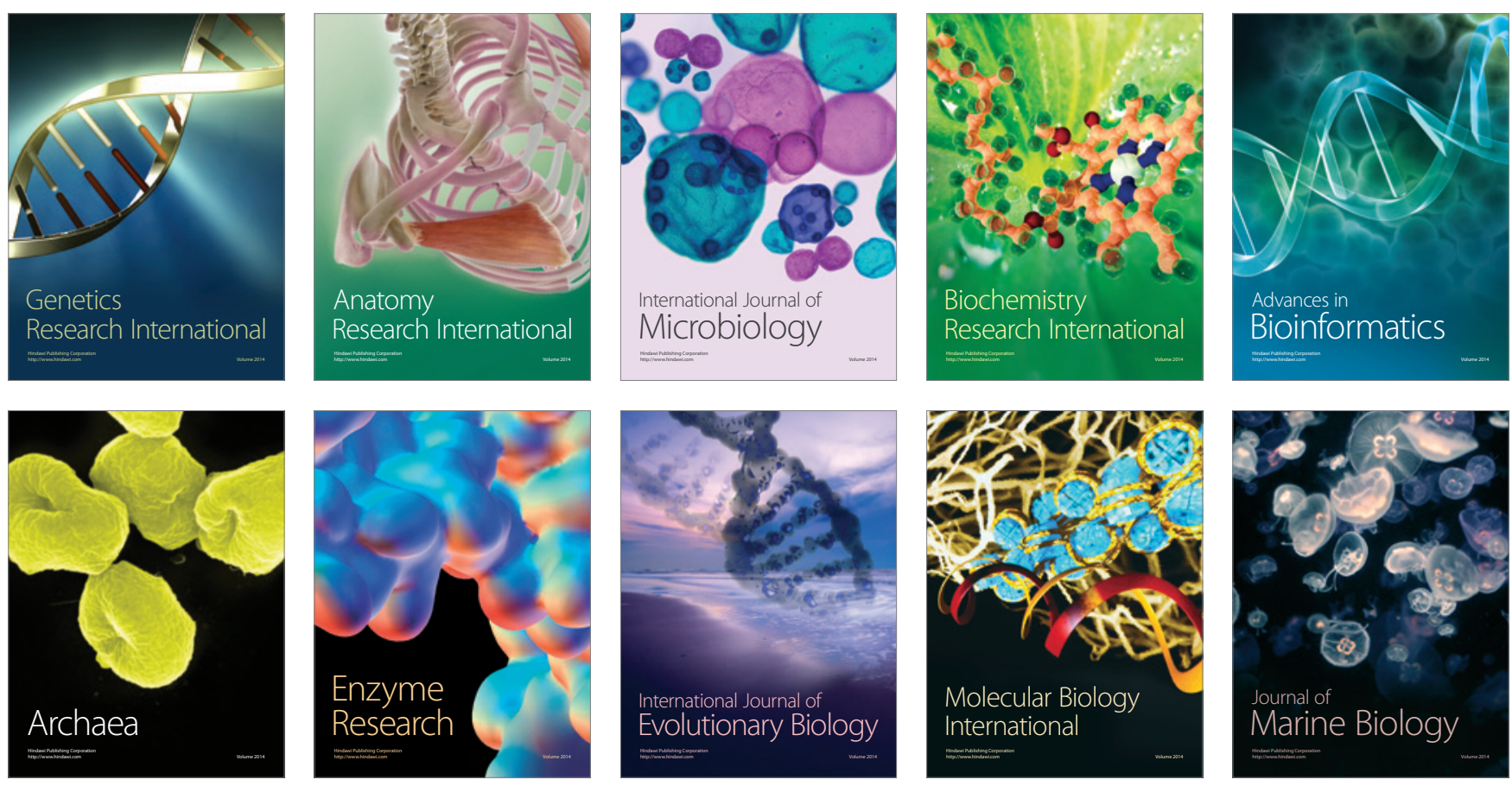